\title{
Prevalence of genetic differences in phosphorylcholine expression between nontypeable Haemophilus influenzae and Haemophilus haemolyticus
}

\author{
Kirk W McCrea ${ }^{1 *}$, Jingping Xie ${ }^{2,3}$, Carl F Marrs², Janet R Gilsdorf ${ }^{1}$
}

\begin{abstract}
Background: Although non-typeable (NT) Haemophilus influenzae and Haemophilus haemolyticus are closely related human commensals, H. haemolyticus is non-pathogenic while NT H. influenzae is an important cause of respiratory tract infections. Phase-variable phosphorylcholine (ChoP) modification of lipooligosaccharide (LOS) is a NT $H$. influenzae virulence factor that, paradoxically, may also promote complement activation by binding C-reactive protein (CRP). CRP is known to bind more to ChoP positioned distally than proximally in LOS, and the position of ChoP within LOS is dictated by specific licD alleles (designated here as lic $D_{l,}$ lic $D_{\| l l}$ and lic $D_{N V}$ ) that are present in a lic1 locus. The lic1 locus contains the licA-licD genes, and ChoP-host interactions may also be influenced by a second licl locus that allows for dual ChoP substitutions in the same strain, or by the number of licA gene tetranucleotide repeats (5'-CAAT-3') that reflect phase-variation mutation rates.

Results: Using dot-blot hybridization, $92 \%$ of 88 NT H. influenzae and $42.6 \%$ of 109 H. haemolyticus strains possessed a lic1 locus. Eight percent of NT H. influenzae and none of the H. haemolyticus strains possessed dual copies of lic1. The lic $D_{I I \mid}$ and lic $D_{I V}$ gene alleles were distributed similarly (18-22\%) among the NT H. influenzae and H. haemolyticus strains while licD, alleles were present in $45.5 \%$ of NT H. influenzae but in less than $1 \%$ of H. haemolyticus strains $(P<.0001)$. NT H. influenzae had an average of 26.8 tetranucleotide repeats in licA compared to14.8 repeats in $H$. haemolyticus $(P<.05)$. In addition, NT H. influenzae strains that possessed a lic $D_{I I}$ allele had increased numbers of repeats compared to NT H. influenzae with other licD alleles $(P<.05)$.

Conclusions: These data demonstrate that genetic similarities and differences of ChoP expression exist between NT H. influenzae and H. haemolyticus and strengthen the hypothesis that, at the population level, these differences may, in part, provide an advantage in the virulence of NT H. influenzae.
\end{abstract}

\section{Background}

Strains of non-typeable (NT) Haemophilus influenzae asymptomatically colonize the human pharynx, but are also opportunistic pathogens that cause localized respiratory tract infections such as otitis media, pneumonia, bronchitis, sinusitis, and COPD exacerbation $[1,2]$. Bacterial factors that differentiate disease from commensal strains are largely unknown since the population structure of NT $H$. influenzae is genetically

\footnotetext{
* Correspondence: sigmb@umich.edu

'Department of Pediatrics and Communicable Diseases, University of Michigan, Ann Arbor, MI 48109, USA

Full list of author information is available at the end of the article

heterologous [3]. The association of bacterial factors with disease-causing strains can be inferred, however, by comparing the prevalence of genetic traits between epidemiologically defined collections of disease and commensal strains [4-7] or, alternatively, between the pathogenic species and a phylogenetically close but nonpathogenic relative [8-11].

Haemophilus haemolyticus is a phylogenetically close relative of NT $H$. influenzae, but has not been associated with disease $[7,12,13]$. The two species reside in the same host niche, overlap extensively by both taxonomic and phylogenetic analyses $[10,14,15]$, and exchange DNA through natural transformation $[10,13,16]$. Given 
their close relationship, but difference in disease potential, NT $H$. influenzae and $H$. haemolyticus likely possess common genes or genetic traits for commensal growth but differ in genes or traits that facilitate disease [10].

Historically, $H$. haemolyticus has been considered a rarely encountered commensal that was easily differentiated from NT $H$. influenzae by its hemolytic phenotype [17-19]. Recent studies, however, have shown that $20-40 \%$ of isolates in various NT $H$. influenzae collections were miss-classified, and found to be non-hemolytic H. haemolyticus $[7,13]$. These observations suggest that $H$. haemolyticus is significantly more prevalent in the pharynges than previously thought, and that clinical differentiation of the species from throat and sputum samples is inadequate [13]. Therefore, we recently sought to differentiate the species by their relative proportions of selected NT $H$. influenzae virulence genes and observed that a probe made to licA, a NT $H$. influenzae gene necessary for phosphorylcholine (ChoP) modification of LOS, hybridized to $96 \%$ of NT $H$. influenzae isolates and to $42 \%$ of $H$. haemolyticus isolates [10]. The relationship of ChoP expression between NT $H$. influenzae and $H$. haemolyticus is unknown but differences between the species may highlight important roles in NT $H$. influenzae virulence.

In studies addressing NT $H$. influenzae virulence, ChoP-modified LOS has been shown to promote bacterial adherence and invasion of host cells through interaction with the platelet activating factor receptor, to increase bacterial resistance to host antimicrobial peptides such as cathelicidin (or LL-37/hCAP18), and to modulate the host inflammatory response directed toward bacteria present in biofilms [20-22]. Paradoxical to its role in enhancing colonization and virulence, ChoP can bind C-reactive protein (CRP) which initiates $\mathrm{C} 1 \mathrm{q}$ binding that leads to activation of the classical complement pathway and bactericidal killing [23]. The concentration of CRP (in both serum and respiratory tract secretions) dramatically increases during inflammation, and has been proposed to facilitate clearance of ChoP-expressing bacteria in the respiratory tract $[24,25]$. Human ChoP-specific antibodies capable of eliciting in vitro bactericidal activity against some $H$. influenzae strains have also been identified, suggesting a further liability of $H$. influenzae ChoP expression [26]. H. influenzae may avoid CRP and anti-ChoP antibody binding, however, by phase varying ChoP expression and by strain-dependent localization of ChoP substitutions within LOS $[27,28]$.

In $H$. influenzae, ChoP expression is controlled by a contingency locus, lic1, that contains the $l i c A, l i c B, l i c C$, and $l i c D$ genes (encoding a choline kinase, a choline permease, a pyrophosphorylase, and a diphosphonucleoside choline transferase, respectively) [29]. Contingency loci, such as lic1, contain simple sequence repeats (SSR) that provide an organism with the ability to phase vary specific phenotypes in response to host challenges [27]. In lic1, the SSR are tetranucleotide (5'-CAAT-3') and are present at the $5^{\prime}$ end of $\operatorname{lic} A$, the first gene in the locus [29]. During replication, intragenic SSR repeats undergo slipped-strand mispairing which results in translational phase variation, and the rate of these mutations is proportional to the length of the repeat region [30]. De Bolle et al [31] found that mutation rates of a $H$. influenzae type III restriction modification gene ( mod) engineered to contain 17-38 tetranucleotide (AGTC) intragenic repeats increased linearly with the number of repeats. In contrast, the same gene containing 5-11 repeats demonstrated rare, if any, phase-variation. Thus, higher numbers of repeats in a contingency locus may protect the bacteria by decreasing the response time to host challenges [27]. Among $H$. influenzae strains, however, the number of licA gene 5'CAAT-3' repeats range from 3-56, and patterns pertaining to virulence have not been identified [32,33].

Depending on the $H$. influenzae strain, ChoP may be substituted at different positions within LOS. Substitutions may occur on oligosaccharides that extend from any one of the three conserved inner-core heptose residues (heptose I, II, and III) or, alternatively, directly to heptose IV, an outer core heptose that extends from heptose I $[34,35]$. These substitutions are dictated largely by the diphosphonucleoside choline transferase encoded by the $l i c D$ gene. Three $l i c D$ gene alleles mediate ChoP substitutions at different positions within LOS and, for simplification, we have named the alleles to reflect their association with a given heptose-residue: lic $D_{I}$, lic $D_{I I I}$, and $l i c D_{I V}$. Although ChoP has been associated with heptose II residues in selected strains, a specific licD allele mediating these substitutions has not been experimentally documented [35]. The deduced LicD proteins are 265-268 amino acids in length and range in sequence identity from $74-88 \%$ with much of the variation occurring in the central part of the primary structure [28,35]. Although most NT H. influenzae strains possess a single $l i c D$ allelic gene that facilitates one ChoP substitution, Fox et al [35] recently reported that $4 / 25$ (16\%) of NT $H$. influenzae middle ear strains possessed two different licD alleles, each present in a separate, phase-variable lic1 locus, that together could produce up to two ChoP substitutions in the strain's LOS.

Both the number and position of ChoP substitutions within LOS may affect binding of host clearance molecules such as CRP or natural ChoP antibodies [26,28]. For instance, $H$. influenzae strains with dual ChoP substitutions bind more CRP, and $H$. influenzae strains with ChoP substitutions positioned from the distal heptose 
III residue are 10-fold more sensitive to CRP-initiated bactericidal killing than ChoP associated with the proximal heptose I in the same strains [28,35]. Consequently, strains with proximal ChoP substitutions (i.e. heptose I) may be more protected from CRP-mediated clearance, and LOS structural studies on selected NT H. influenzae strains have found that ChoP predominate at this position [34]. The overall prevalence of these substitutions in the NT $H$. influenzae population, however, is not known. Differences in the prevalence of single or combined licD gene alleles between NT $H$. influenzae and $H$. haemolyticus may reflect the importance of ChoP structures in NT $H$. influenzae virulence.

The presence of a licA gene in $H$. haemolyticus suggests that it may contain a lic1 locus and express ChoP in a manner similar to $H$. influenzae [10]. Since ChoP expression among NT $H$. influenzae strains can vary greatly due to genetic factors listed above, we speculated that differences in the prevalence of these factors between strain populations of $H$. influenzae and $H$. haemolyticus may highlight, in part, which ones provide an advantage to $H$. influenzae in transcending from commensal to disease-related growth.

\section{Results}

\section{ChoP expression in $H$. haemolyticus}

Although $H$. influenzae is known to modify its LOS with ChoP, expression and surface localization of ChoP in $H$. haemolyticus has not been demonstrated. To investigate ChoP expression in $H$. haemolyticus, we obtained LOS profiles on silver-stained tricine SDS-PAGE from wholecell lysates on three $H$. influenzae control strains, six $H$. haemolyticus strains containing a $\operatorname{lic} A$ gene, and five $H$. haemolyticus strains lacking a licA gene [10]. As seen in Figure 1 (upper panel), both NT $H$. influenzae and $H$. haemolyticus demonstrated intra-and inter-strain variability in LOS migration. A duplicate gel was transferred to a Western immunoblot and ChoP was detected with TEPC-15, a mAb that recognizes ChoP on a number of pathogenic bacteria [36-38]. TEPC-15 reacted with LOSassociated bands in all $H$. influenzae control strains and in the six $H$. haemolyticus strains that contained a licA gene (Figure 1 lower panel). The antibody, however, did not react to five $H$. haemolyticus strains lacking a licA gene (Figure 1 lower panel).

The association of ChoP epitopes with $H$. haemolyticus LOS was further supported by proteinase $\mathrm{K}$ digestion experiments. TEPC-15 reactivity was still present on Western immunoblots containing $H$. influenzae strain $\mathrm{Rd}$ and $H$. haemolyticus strain M07-22 that were pre-treated with proteinase $K$, although no proteins were visible in these preparations when they were run on glycine SDS-PAGE and stained with Coomassie (data not shown). Together these results suggest that, similar

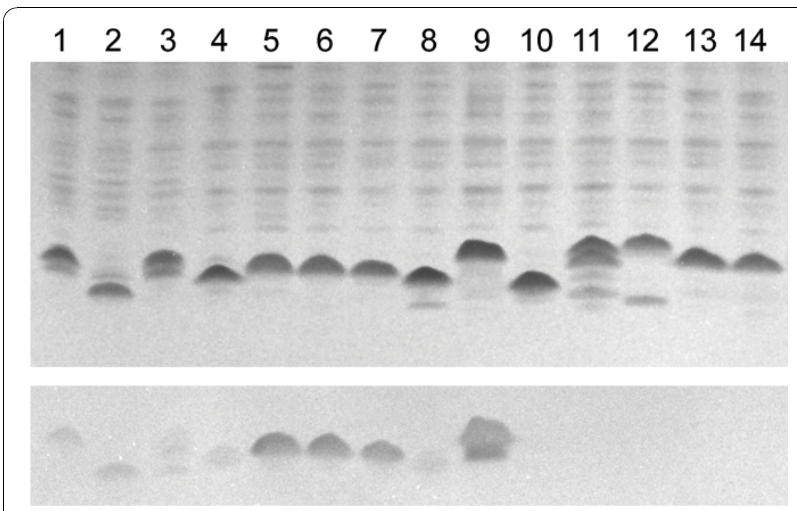

Figure 1 LOS profiles and TEPC-15 mAb reactivity in H. haemolyticus. H. influenzae and H. haemolyticus whole-cell lysates were run on tricine SDS-PAGE and silver stained to visualize LOS migration (upper panel) or transferred to nitrocellulose membrane for reactivity with the ChoP-specific mAb, TEPC-15

(lower panel). Lanes 1-3, H. influenzae ChoP phase-on variant strains (E1a, Rd, and Mr15); lanes 4-9, H. haemolyticus strains hybridizing with a licA gene probe $(\mathrm{M} 07-22,60 \mathrm{P} 3 \mathrm{H} 1,7 \mathrm{P} 24 \mathrm{H}, 3 \mathrm{P} 41 \mathrm{H} 5, \mathrm{C} 03-22$, and H01-21); and lanes 10-14, H. haemolyticus strains not hybridizing with a licA gene probe (ATCC 33390, 3P18H1, 24P4 H, 26428, 26322).

to $H$. influenzae, some strains of $H$. haemolyticus can express a ChoP epitope that is localized within its LOS.

\section{H. haemolyticus contains a lic1 locus similar to H. influenzae}

The ability of $H$. haemolyticus to hybridize with a $H$. influenzae licA gene probe suggests that $H$. haemolyticus contains a lic1 locus [10]. In $H$. haemolyticus strains M07-22 and 60P3H1, licA-licD gene probes were each found to hybridize with one restriction fragment on Southern blots, suggesting that all genes were confined to a single locus in each strain (data not shown). PCR designed to amplify overlapping regions of $H$. influenzae lic1 locus genes also amplified similar products in $H$. haemolyticus strains M07-22 and 60P3H1, and DNA sequencing of these products revealed that the strains contained tandem licA-licD genes spanning 3375 and 3324 bp [GenBank: HM140372 and HM140373], respectively, similar in size to the lic1 loci present in the genome-sequenced $H$. influenzae strains $\mathrm{Rd}(3358 \mathrm{bp})$ and 86-028NP (3333 bp) [39,40]. Further comparisons of the lic1 loci between $H$. haemolyticus and $H$. influenzae [29] revealed that, in both species, the loci were flanked by the same chromosomal genes, contained lic $A \alpha, \beta$, and $\gamma$ start codons positioned immediately upstream of tandemly arranged tetranucleotide (5'-CAAT-3') repeats, and contained $l i c B$ and $l i c C$ start codons that overlapped each preceding gene (data not shown).

The LicA, LicB, and LicC amino-acid sequences for the two $H$. haemolyticus strains M07-22 and 60P3H1 were deduced and found to be 93, 99, and 95\% identical, 
Table 1 Amino-acid sequence identities between the LicA-LicD proteins of $H$. influenzae and $\boldsymbol{H}$. haemolyticus

\begin{tabular}{lcccccccc}
\hline & \multicolumn{3}{c}{ LicA } & \multicolumn{3}{c}{ LicB } & \multicolumn{3}{c}{ LicC } & \multicolumn{2}{c}{ LicD } \\
\hline Strains & M07-22 & $60 \mathrm{P} 3 \mathrm{H} 1$ & M07-22 & $60 \mathrm{P} 3 \mathrm{H} 1$ & M07-22 & $60 \mathrm{P} 3 \mathrm{H} 1$ & M07-22 & $60 \mathrm{P} 3 \mathrm{H} 1$ \\
E1a & 87.2 & 86.9 & 92.8 & 93.5 & 89.7 & 89.3 & 94.8 & 68.7 \\
Rd & 86.9 & 86.9 & 93.2 & 93.8 & 92.7 & 92.3 & 89.4 & 69.4 \\
86-028NP & 86.9 & 86.9 & 89.7 & 90.1 & 89.7 & 89.3 & 87.2 & 68.3 \\
60P3H1 & 93.3 & & 99.3 & & 94.8 & & 69.1 & \\
\hline
\end{tabular}

respectively, between the strains (Table 1). Amino-acid sequences comparisons of the putative LicA, LicB, and LicC proteins between $H$. haemolyticus and $H$. influenzae (strains E1a, $\mathrm{Rd}$, and 86-028NP) revealed identities that were somewhat lower, ranging from $87-94 \%$ for all comparisons (Table 1). As mentioned above, three LicD protein alleles $\left(\mathrm{LicD}_{\mathrm{I}}, \mathrm{LicD}_{\mathrm{III}}\right.$, and $\left.\mathrm{LicD}_{\mathrm{IV}}\right)$ have been described for $H$. influenzae. The LicD protein of $H$. haemolyticus strain M07-22 was 89 and $87 \%$ identical to the $\mathrm{LicD}_{\mathrm{I}}$ allele of $H$. influenzae strains $\mathrm{Rd}$ and 86-028NP, respectively, but was 95\% identical with and contained a 3 amino-acid insertion similar to the LicD $_{\text {III }}$ allele of $H$. influenzae strain E1a, suggesting that this $H$. haemolyticus strain possessed a LicD III allele (Table 1 ). In contrast, the putative LicD protein of $\mathrm{H}$. haemolyticus strain 60P3H1 averaged only $69 \%$ identity with the LicD alleles of $H$. haemolyticus strain M07-22 and the three $H$. influenzae strains (Table 1). BLAST analysis, however, revealed that it was $99 \%$ identical to the deduced LicD IV protein of NT $H$. influenzae strain R2866, suggesting that $H$. haemolyticus strain $60 \mathrm{P} 3 \mathrm{H} 1$ contained a LicD $\mathrm{IV}_{\text {allele. Together, }}$ these data suggest that $H$. haemolyticus possess lic1 loci that are very similar to the lic1 loci described for H. influenzae.
Prevalence of lic 1 loci in $\boldsymbol{H}$. influenzae and $\boldsymbol{H}$. haemolyticus As mentioned, the prevalence of the $l i c A$ gene has been reported for a phylogenetically defined NT $H$. influenzae and $H$. haemolyticus strain collection [10]. We therefore determined the distribution of the remaining lic1 locus genes (licB, lic $C$, and $l i c D$ ) among the same strains by dot-blot hybridization. The licB-licD gene probes each hybridized to three $H$. influenzae positive control strains (Rd, 86-028NP, and R2866), to 81/88 (92\%) NT H. influenzae strains and to $46 / 109$ (42.2\%) H. haemolyticus strains. Four NT $H$. influenzae strains (53122, B01-21, $\mathrm{H} 08-25$, and $\mathrm{H} 10-21)$ that previously hybridized with the licA gene probe did not hybridize with the licB-licD probes. In addition, one NT $H$. influenzae strain (32324) that did not previously hybridize with the licA gene probe did hybridize with the licB-licD probes in this study. Repeat hybridization of these discrepant strains with the $\operatorname{lic} A$ gene probe revealed that $l i c A$ hybridization was concordant with $l i c B$-licD hybridization, and that all strains either lacked or possessed all four lic1 locus genes. The probes did not hybridize to a negative control species ( $N$. meningitidis) or to any of the remaining NT $H$. influenzae or $H$. haemolyticus strains that previously failed to hybridize with the licA gene probe (Table 2). The absence of the licA-licD genes in these

Table 2 Prevalence of lic1 locus copy number and licD alleles in NT $\boldsymbol{H}$. influenzae and $\boldsymbol{H}$. haemolyticus

\begin{tabular}{|c|c|c|c|c|}
\hline Genotype & H. influenzae $\mathrm{n}=88(\%)$ & H. haemolyticus $\mathrm{n}=109(\%)$ & $\mathrm{PR}^{\mathrm{a}}$ & $P$ value ${ }^{c}$ \\
\hline \multicolumn{5}{|c|}{ lic1 copy number } \\
\hline 0 & $7(8.0)$ & $63(57.8)$ & 0.14 & $<.0001$ \\
\hline 1 & $74(84.0)$ & $46(42.2)$ & 2.18 & $<.0001$ \\
\hline 2 & $7(8.0)$ & $0(0)^{b}$ & ND & .0031 \\
\hline \multicolumn{5}{|l|}{ single licD alleles } \\
\hline licD, & $40(45.5)$ & $1(0.92)$ & 49.5 & $<.0001$ \\
\hline$\|_{i c D_{I I}}$ & $14(15.9)$ & $23(21.1)$ & 0.75 & .6647 \\
\hline $\operatorname{lic} D_{I V}$ & $20(22.7)$ & $23(21.1)$ & 1.07 & .3536 \\
\hline \multicolumn{5}{|l|}{ dual licD alleles } \\
\hline $\operatorname{lic}_{I V}-$ lic $_{I I I}$ & $4(4.5)$ & $0(0)$ & ND & .0383 \\
\hline licD, $_{\text {rlicD }}|| l$ & $1(1.1)$ & $0(0)$ & ND & .4467 \\
\hline $\operatorname{lic}_{1}{ }_{r}$ lic $D_{N}$ & $1(1.1)$ & $0(0)$ & ND & .4467 \\
\hline licD, licD, & $1(1.1)$ & $0(0)$ & ND & .4467 \\
\hline
\end{tabular}

\footnotetext{
a Prevalence ratios (PR) were calculated for $H$. influenzae using $H$. haemolyticus as the referent group.

${ }^{\mathrm{b}}$ Logit, 0.5 used in place of 0 for PR and statistical calculations.

${ }^{c} P<0.05$ is considered statistically significant using $\chi^{2}$ analysis.
} 
strains suggests that $8 \%$ of NT H. influenzae and $57.8 \%$ of $H$. haemolyticus strains lack a lic1 locus for ChoP expression, and that absence of a lic1 locus is 7.23 times more prevalent in $H$. haemolyticus than in NT $H$. influenzae (expressed in Table 2 as 0.14 times prevalent for NT $H$. influenzae, $P<.05)$.

The prevalence of NT $H$. influenzae and $H$. haemolyticus strains possessing single or duplicate lic1 loci is not known. Similar to the method reported by Fox et al [35], we screened our 81 NT H. influenzae and $46 H$. haemolyticus lic1-containing strains for duplicate lic1 loci using Southern hybridization of Mfe 1 digested genomic DNA to identify two restriction fragments that hybridized with a $l i c D$ gene probe. Strains with two licD-hybridizing bands were present in seven NT $H$. influenzae strains and in none of the $H$. haemolyticus strains. Further hybridization using a licA gene probe on the seven NT $H$. influenzae strains also revealed two licA hybridizing bands in these strains, suggesting that they possessed two complete lic1 loci. Assessing the population prevalence of licl locus copy number among the species, the data suggest that 74/88 (84\%) NT $H$. influenzae and 46/109 (42.2\%) H. haemolyticus possess one copy of lic1, and that strains with one lic1 locus are 2.18 times more prevalent in NT $H$. influenzae than in H. haemolyticus $(P<.0001)$ (Table 2). Duplicate lic1 loci were present in 7/88 (8\%) NT H. influenzae and 0/ 109 (0\%) H. haemolyticus strains, suggesting that duplicate lic1 loci in H. haemolyticus are rare or altogether absent (Table 2).

\section{Prevalence of the three LicD alleles in NT $H$. influenzae and $H$. haemolyticus}

Determining the prevalence of the three previously described $l i c D$ alleles among the two species was initiated by PCR amplification and DNA sequence analysis of the licD genes from the 74 NT $H$. influenzae and $46 \mathrm{H}$. haemolyticus strains in our collection that contained a single lic1 locus. The deduced LicD aminoacid sequences of these strains were determined [GenBank:HM133649-HM133768] and the licD gene from one NT $H$. influenzae strain (Mr27) was repeatedly found to possess a nonsense mutation that would result in gene termination. A minimum-evolution dendrogram (in radiation view) was created from the remaining $\mathrm{LicD}$ amino-acid sequences of the NT $H$. influenzae and $H$. haemolyticus strains. The dendrogram revealed three distinct clusters, each containing a different $H$. influenzae prototype $\mathrm{LicD}$ allele $\left(\mathrm{LicD}_{\mathrm{I}}\right.$ from strains $\mathrm{Rd}$ and 86023NP, Lic $\mathrm{D}_{\text {III }}$ from strain $\mathrm{E} 1 \mathrm{a}$, and $\mathrm{Lic}_{\mathrm{IV}}$ from strain R2866) (Figure 2). These results suggest that the three previously defined LicD alleles represent the major allelic variants found among the $H$. influenzae and $H$. haemolyticus species.

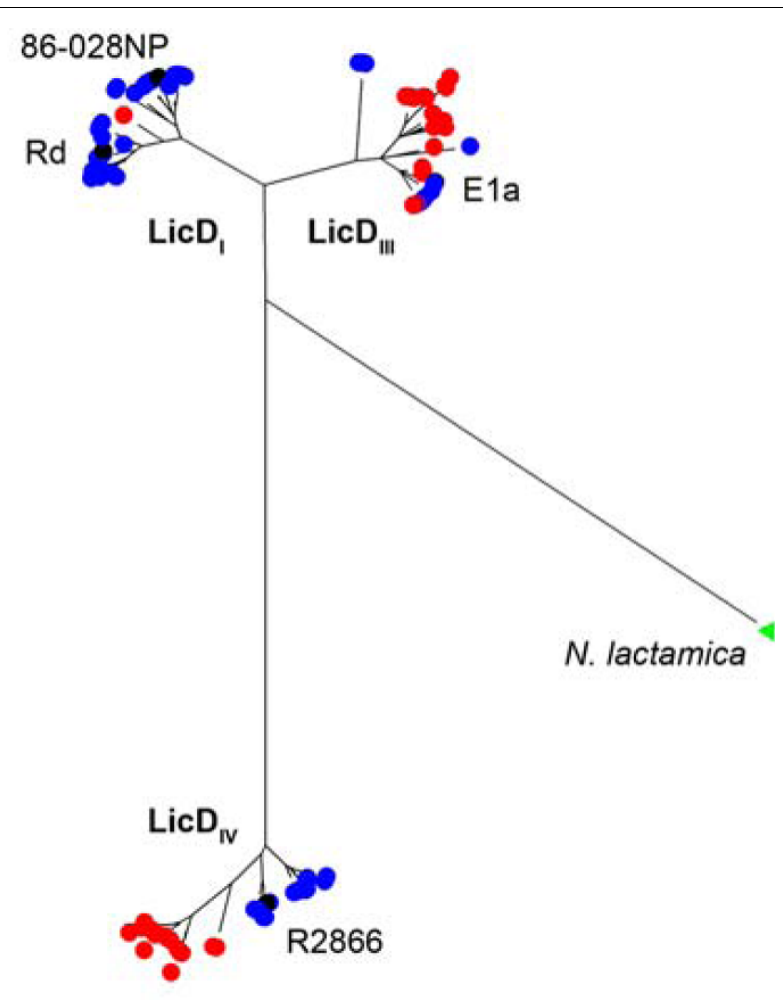

Figure 2 Clustering of $\boldsymbol{H}$. influenzae and $\boldsymbol{H}$. haemolyticus LicD alleles. The major clusters of $\mathrm{H}$. influenzae (blue dots) and $\mathrm{H}$. haemolyticus (red dots) strains are labeled by their predicted allele ( $\mathrm{LiCD}_{\text {I, }} \mathrm{LiCD}_{\mathrm{III}}$, and LicD $\mathrm{IV}_{\mathrm{V}}$ ) and prototype LicD alleles from $\mathrm{H}$. influenzae strains are shown for each cluster (black dots, Ela is partially hidden). The LicD protein of N. lactamica is the out-group for the analysis (green triangle).

Next, we determined the population prevalence of specific licD alleles in our NT H. influenzae and $H$. haemolyticus strains. Among the 88 total NT H. influenzae strains in the collection, $43(49 \%)$ possessed a single lic $D_{I}$ allele, 19 (22\%) possessed a single $l i c D_{I I I}$ allele, and $25(28 \%)$ possessed a single $l i c D_{I V}$ allele (Table 2). In contrast, only 1 of the $109(0.9 \%) H$. haemolyticus strains possessed a $l i c D_{I}$ allele while $23(21 \%)$ possessed a single $l i c D_{I I I}$ allele and $23(21 \%)$ possessed a single lic$D_{I V}$ allele. Although the prevalence of single $l i c D_{I}$ alleles was statistically different between NT $H$. influenzae and $H$. haemolyticus $(P<.0001)$, the prevalence of the lic $D_{I I I}$ and $l i c D_{I V}$ alleles was not statistically different between the species (Table 2). Assessment of $l i c D$ gene alleles among the seven dual lic1 locus-containing NT H. influenzae strains was determined by PCR amplifying and sequencing licD from agarose gel slices of strain DNA digested with $M f e 1$. The results revealed that $4 / 88$ $(4.5 \%)$ strains had $l i c D_{I I I}$-lic $D_{I V}$ alleles, while only $1 / 88$ (1.1\%) strains each were found to possess combinations of lic $D_{I^{-}}-l i c D_{I I I}$, lic $D_{I^{-}} l i c D_{I V}$, and $l i c D_{I^{-}}$lic $D_{I}$ alleles (Table 2). Together, these results suggest that the $\operatorname{lic} D_{I}$ 
allele is rarely present in $H$. haemolyticus, and that the proportions of $l i c D_{I I I}$ and $l i c D_{I V}$ alleles are similar between the species.

\section{ChoP phase variation and the number of licA tetranucleotide (5'-CAAT-3') repeats among NT $H$. influenzae and $\boldsymbol{H}$. haemolyticus}

Phase variation of ChoP expression is similar between NT $H$. influenzae and $H$. haemolyticus. The licA genes of $H$. haemolyticus strains M07-22 and 60P3H1 contained a number of 5'-CAAT-3' repeats that would place the $\operatorname{lic} A$ gene in a correct translational open reading frame (data not shown). ChoP expression in these two strains was corroborated by Western immunoblot where TEPC-15 reactive epitopes were present in each strain (Figure 1, lanes 4 and 5). In addition, phase-negative variants could be isolated from each $H$. haemolyticus strain, and DNA sequence analysis revealed that each licA repeat region gained one 5'-CAAT-3' repeat, placing the licA gene out of frame (data not shown).

Mutation rates in contingency loci are proportional to the length of the repeat region in the loci and the repeat region length may therefore affect the ability of bacteria to respond to a host immunologic challenge [31]. To determine if a general population difference of $\operatorname{lic} A$ repeat length exists between the species in this study, we compared the number of licA 5'-CAAT-3' repeats between the 74 NT $H$. influenzae and $46 \mathrm{H}$. haemolyticus strains that contained a single lic1 locus. DNA sequence analysis of PCR amplified repeat regions from these strains revealed a wide range in repeat numbers for both species (5-45 and 6-56 repeats for NT $H$. influenzae and $H$. haemolyticus, respectively) (Figure 3, Table $3)$. The average number of $l i c A$ repeats between the species, however, was statistically different with NT $H$. influenzae having a mean of 27 repeats and $H$. haemolyticus having a mean of 15 repeats $(P<.0001$ using the student's T test) (Table 3 ). These results suggest that, at the population level, the contingency response for ChoP expression may be slower for $H$. haemolyticus than for NT $H$. influenzae.

H. influenzae strains that express ChoP at more distal positions in LOS (i.e. ChoP substituted on an oligosaccharide extending from heptose III) have been shown to be more vulnerable to CRP binding, leading to activation of complement mediated bactericidal killing [28]. Therefore, strains may differ in their licA mutation rates depending on which LOS structure is modified with ChoP. To test this, we further stratified the number of lic $A$ gene repeats between strains with different $l i c D$ alleles for each species. Among NT $H$. influenzae, the range of repeats was similar among strains that possessed a $l i c D_{\mathrm{I}}, l i c D_{I I I}$, or $l i c D_{I V}$ allele $(6-45,5-43$, and 9-42 repeats, respectively) (Table 3 ). The average number of repeats was significantly different, however, for strains that possessed a $l i c D_{I I I}$ allele (34 repeats) than for strains that possessed a $l i c D_{\mathrm{I}}$ or $l i c D_{I V}$ allele $(25$ and 26 repeats, respectively) ( $P=.015$ and .032 using the student's $\mathrm{T}$ test, respectively) (Table 3 ). Among $H$. haemolyticus, the range of $l i c A$ repeats was more variable between strains with $l i c D_{I I I}$ and $l i c D_{I V}$ alleles (6-56 and 6-27 repeats, respectively), due mainly to three $l i c D_{I I I^{-}}$ containing strains with licA genes that contained 39, 40, and 56 repeats (Table 3 , Figure 3 ). In contrast to NT $H$. influenzae, however, the average number of repeats was not significantly different between $H$. haemolyticus strains possessing $l i c D_{I I I}$ or $l i c D_{I V}$ alleles (16 and 13, respectively) (Table 3 ). These results suggest that NT $H$. influenzae strains that substitute ChoP on more proximal, exposed oligosaccharides chains may tend to have increased mutation rates within the repeat region of the lic $A$ gene.

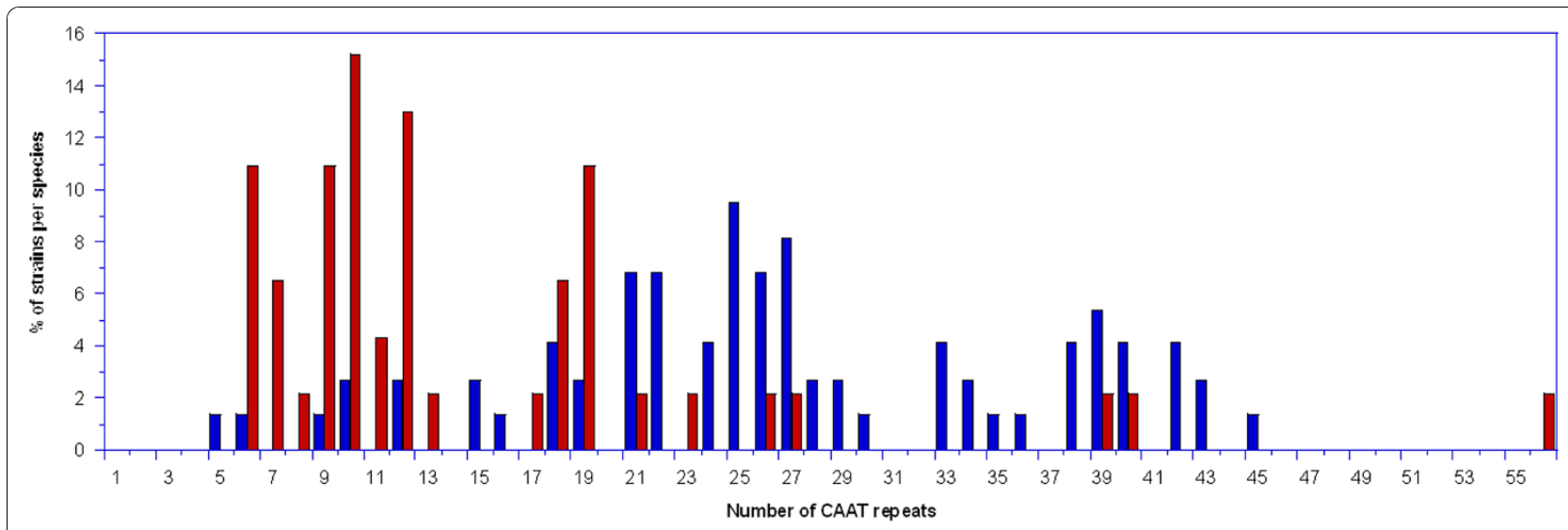

Figure 3 Distribution of NT $\boldsymbol{H}$. influenzae and $\boldsymbol{H}$. haemolyticus strains with various numbers of CAAT repeats. Percent of lic1-positive NT $H$. influenzae and $H$. haemolyticus strains based on the number of CAAT repeats they contain. NT H. influenzae and $H$. haemolyticus are labeled in blue and red, respectively. 
Table 3 Stratification of the number of licA gene 5'CAAT-3' repeats between species and licD alleles

\begin{tabular}{lccc}
\hline Stratification & Strains $(\mathbf{n})$ & Range & Average \pm S.D. \\
\hline Species & & & \\
$\quad$ NT H. influenzae & 74 & $5-45$ & $27 \pm 10^{*}$ \\
$\quad$ H. haemolyticus & 46 & $6-56$ & $15 \pm 4$ \\
NT H. influenzae licD alleles & & & \\
$\quad$ licD & 40 & $6-45$ & $25 \pm 9$ \\
$\quad$ lic $D_{I I I}$ & 14 & $5-43$ & $34 \pm 11^{* *}$ \\
$\quad$ licD & 20 & $9-42$ & $26 \pm 8$ \\
H. haemolyticus licD alleles & & & \\
$\quad$ lic $D_{I I I}$ & 23 & $6-56$ & $16 \pm 13$ \\
$\quad$ licD & 23 & $6-27$ & $13 \pm 6$
\end{tabular}

${ }^{*} P<.0001$ using the student's T-test

** $P<.05$ for each comparison using the student's T-test

\section{Discussion}

The strain population structure of NT $H$. influenzae is genetically very diverse and clones or clusters of NT $H$. influenzae strains that differentiate virulent from commensal strains have not been identified [10,41]. Given this diversity, together with the high prevalence of NT $H$. influenzae colonization in the healthy human population, it is reasonable to hypothesize that not all NT $H$. influenzae strains possess the same ability to cause disease, but rather, that a proportion of strains possess a range of variable genetic traits that allow for infection and disease under the right host conditions [42]. Thus, comparison of genetic trait prevalence between populations of NT $H$. influenzae and the closely related but strictly commensal species, $H$. haemolyticus, will highlight traits within the species' gene pools that may offer clues to the virulence pathways of NT $H$. influenzae. For instance, ChoP expression in NT $H$. influenzae is strongly implicated as a virulence factor $[43,44]$ and is thought to enhance virulence though increased epithelial cell adherence, inhibition of bactericidal peptides, and modulation of the immune system during biofilm growth [20-22]. In this study, 58\% of $H$. haemolyticus strains lacked a lic1 locus (and the ability to express ChoP) while only $8 \%$ of NT $H$. influenzae strains lacked a lic1 locus, suggesting that, at a population level, ChoP expression may provide an advantage for more NT $H$. influenzae strains to cause disease. Furthermore, the trend of shorter licA gene repeat regions in $H$. haemolyticus strains that possess a lic1 locus (and the potential to express ChoP), may suggest that those strains have a slower phase-variable response to host defences targeting ChoP (i.e. CRP), potentially limiting their survival in inflammatory environments. Obviously, prevalence differences in ChoP expression alone do not account for all differences in disease potential between the species since many other virulence factors have been described for NT H. influenzae. Rather, the differential prevalence of genetic traits between the species highlight factors that may be further studied for their roles in virulence using in vitro and in vivo models of NT H. influenzae infection.

Although the structure of $H$. haemolyticus LOS is unknown, the assumption has been made that basic LOS structures and biosynthesis of ChoP modifications, mediated by the phosphocholine transferase, LicD, are comparable between NT $H$. influenzae and H. haemolyticus. Some evidence suggests that these assumptions are reasonable. In the tricine SDS-PAGE experiments of this study, $H$. haemolyticus LOS migrated at a rate similar to the LOS of NT $H$. influenzae, and $H$. haemolyticus LOS also presented intra and inter-strain structural heterogeneity similar to the LOS of NT $H$. influenzae (Figure 1). Recent structural analysis on the LOS of Haemophilus parainfluenzae, a member of the Pasteurellaceae family that is phylogenetically more distant to NT $H$. influenzae than $H$. haemolyticus, revealed that the inner core structure was nearly identical to that of NT H. influenzae [45]. Furthermore, the LicD $\mathrm{D}_{\mathrm{III}}$ and Lic$\mathrm{D}_{\mathrm{IV}}$ alleles of the two $H$. haemolyticus strains in this study demonstrated higher sequence identity (95-99\%) to their cognate proteins in NT $H$. influenzae than similar comparisons of LicA, LicB, and LicC proteins (87$94 \%$, Table 1), suggesting a functional equivalence of the LicD protein alleles. Although these observations are circumstantial, they argue for more detailed comparisons of LOS structures between NT $H$. influenzae and $H$. haemolyticus to identify dissimilarities between the structures that may be associated with the ability of NT $H$. influenzae to cause disease.

The results of this study suggest that genotypes facilitating LOS-ChoP structures that are not conducive to CRP binding predominate among the strain populations of both species; the majority of $H$. haemolyticus strains (58\%) lacked a lic1 locus (indicating no ChoP expression) and the majority of NT $H$. influenzae strains either lacked a lic1 locus or possessed a single $l i c D_{I}$ allele (an allele known to dampen CRP binding by positioning ChoP substitutions from the proximal inner core heptose) (54\% total strains). In comparison, strains possessing single $l i c D_{I I I}$ and $l i c D_{I V}$ alleles were in smaller, but similar fractions in the strain populations of both species (16-23\%), indicating that these allelic distributions are still maintained in the species despite possible increased vulnerability to CRP binding. Further studies that assess the prevalence of licD alleles between epidemiologically comparable collections of virulent and commensal NT $H$. influenzae strains may highlight which alleles are important in NT $H$. influenzae disease.

One ChoP genotype that may be associated with NT $H$. influenzae disease isolates is the possession of two 
lic1 loci in the same strain where each locus contains a different $l i c D$ allele, providing the bacteria with two independently phase-variable ChoP substitutions. Fox et al [35] demonstrated that 4/25 (16\%) NT H. influenzae middle ear strains had dual lic1 loci. In the current study, only NT $H$. influenzae and not $H$. haemolyticus possessed dual lic1 loci. Although only 7 of 88 (8\%) total NT $H$. influenzae strains had dual loci, six were present among 43 (14\%) middle ear strains present in this collection (unpublished results). Fox et al. [35] also noted that the genome sequenced NT $H$. influenzae strain, R2846, possessed a complete and partial lic1 loci, each containing a different $l i c D$ allele, raising the possibility that other strains may have a similar genotype. An extensive search on the lic1-containing strains in this collection using licD-specific PCR and hybridization, however, did not identify any strains (apart from the seven dual lic1 locus strains) that contained more than one licD allele, suggesting that the NT $H$. influenzae population contains mainly complete copies of lic1 (unpublished results).

Although NT $H$. influenzae LOS structural studies have identified ChoP modifications on oligosaccharides extending from the heptose II position [46], specific licD alleles mediating this arrangement have not been characterized. It is possible that one or more of the current LicD alleles may overlap in this process or that stochastic factors in LOS biosynthesis may play a role. In addition, the clustering analysis of LicD protein alleles present in Figure 2 suggests that sub-variants may exist within the major allelic groups, and it is possible that one of these variants may facilitate heptose II-associated ChoP substitutions.

As reviewed by Moxon et al [27], strains that are genetically and epidemiologically unrelated vary widely in the lengths of SSR (including lic $A$ tetranucleotide repeats), while individual strains that transmit within an outbreak or are extensively subcultured over time maintain a central modality in repeat numbers [32,33]. Using a larger number of samples from a phylogenetically defined collection of NT $H$. influenzae strains has allowed us to partially resolve distribution trends for the licA repeat region in the NT $H$. influenzae and $H$. haemolyticus populations (Figure 3) and make statistical comparisons between and within species (Table 3). We found statistically significant trends toward the increased length of licA tetranucleotide repeats in NT H. influenzae compared to $H$. haemolyticus, and in NT H. influenzae strains with $l i c D_{I I I}$ alleles compared to NT $H$. influenzae strains with $l i c D_{\mathrm{I}}$ or $l i c D_{I V}$ alleles. Longer repeat regions are predicted to increase lic1 loci mutation rates and ChoP phase variation, providing increased resistance to host clearance mechanisms such as CRP or antibodies that bind ChoP and initiate complement mediated bactericidal killing. The presence of the longest repeat (56 repeats) in a $H$. haemolyticus strain and only five repeats in a lic $D_{I I I}$-containing NT $H$. influenzae strain, however, are reminders that these trends must be considered in the light of numerous other factors that contribute to the commensal life style of both species and disease potential of NT H. influenzae.

\section{Conclusions}

In summary, the lic1 locus is not part of the conserved "core" genome of the $H$. influenzae population but is part of the flexible gene pool that exists among different strains [47]. Nonetheless, the conserved chemical nature of ChoP and the discovery of anti-ChoP antibodies in human serum provides reasonable credence to ChoP as a vaccine candidate that may inhibit $H$. influenzae at some point in the infectious process. Knowledge of how ChoP expression varies both genetically and structurally within the NT $H$. influenzae strain population is critical for designing intervention strategies that will effectively target disease-related strains. Furthermore, contrasting the genetic properties of NT H. influenzae ChoP expression with those of $H$. haemolyticus, a closely related but non-pathogenic species, has highlighted a number of ChoP expression differences (lic1 copy number, licD alleles, and $l i c A$ repeat number) that may provide an advantage to disease-related growth in NT $H$. influenzae.

\section{Methods}

\section{Bacterial strains and culture methods}

For most studies, bacteria were grown on chocolate agar plates (BBL). ChoP expression was carried out on Levinthal agar [48]. All cultures were incubated at $37^{\circ} \mathrm{C}$ with $5 \% \mathrm{CO}_{2}$.

The $88 \mathrm{NT} H$. influenzae and $109 \mathrm{H}$. haemolyticus strains were parts of various collections obtained by this or other laboratories in previous studies [13,49-54]. All clinical and commensal strains in the current study were used with the approval of the University of Michigan Institutional Review Board. These same strains have been previously characterized for their taxonomic and phylogenetic relationships [10]. Reference strains used in this study included the complete or partially genome sequenced $H$. influenzae strains Rd (KW-20, ATCC 51907), 86-028NP [NT nasopharyngeal strain associated with otitis media], R2866 (INT-1, ATCC 51997; a NT, invasive strain), and a $H$. haemolyticus type strain, ATCC 33390. A negative-control species, $N$. meningitidis strain G1723, was used in dot-blot hybridization. Two $H$. haemolyticus strains, M07-22 and 60P3H1, were used to detail the lic1 locus and demonstrate ChoP expression in $H$. haemolyticus. M07-22 is a hemolytic strain obtained from the throat of a healthy child attending day care and $60 \mathrm{P} 3 \mathrm{H} 1$ is a non-hemolytic strain from the sputum of an 
adult with COPD (although not associated with COPD exacerbation) $[10,13]$.

\section{DNA isolation and PCR}

Purified genomic DNA for Southern blots or PCR template was obtained from bacterial strains using the Wizard Genomic DNA purification kit from Promega, Co. (Madison, WI). Oligonucleotides for PCR amplification of gene probes, lic1 loci, and licD alleles were synthesized by Invitrogen and are shown in Table 4 . PCR amplification of the tetranucleotide repeat region was performed as previously described [23] and sequence analysis was done with the primers listed in Table 4. PCR conditions have been described elsewhere [10] and all amplification products were confirmed by $1 \%$-agarose gel electrophoresis.

\section{DNA sequencing}

DNA sequences of the lic1 loci of $H$. haemolyticus strains M07-22 and 60P3H1, the licD allelic genes and the tetranucleotide repeat regions of all strains in the collection possessing licA-licD genes were obtained from PCR products purified on QIAquick columns from Qiagen (Valencia, CA). Automated fluorescent dideoxyDNA sequencing was done by the University of Michigan DNA sequencing core on an ABI model 3730 sequencer. Sequence editing and gene and locus assembly were done with Lasergene software (version 7.0; DNAStar, Madison, WI). Cluster analysis of the LicD protein alleles was done using Mega software (version 3.1) [55]. A bootstrap consensus, minimum-evolution dendrogram of LicD amino-acid sequence was made with 1,000 replicates.

\section{Dot and Southern-blot hybridization}

The bacteria were harvested in PBS to an O.D. ${ }_{600}$ of 1.0, lysed, and frozen as previously described [10]. For dotblot analysis, $40 \mu \mathrm{l}$ of crude lysate DNA obtained from Haemophilus strains grown on chocolate agar was applied in an $8 \times 12$ array on nylon membranes as previously described [10].

PCR-amplified genes were purified from agarose gels using the QIAquick Gel Extraction Kit (Qiagen), and labeled with the AlkPhos Direct ${ }^{\mathrm{Tm}}$ Labeling and Detection System (GE Healthcare, Piscataway, NJ). Probes were hybridized to the dot-blot membranes under stringent conditions and developed by the ECF detection system (GE Healthcare). Probe signal intensity was read by a Storm ${ }^{\text {tw }} 860$ phosphorimager and analyzed with ImageQuant version 5.0 software (Molecular Dynamics/GE Healthcare) [10].

Southern blots to identify lic1 loci in $H$. haemolyticus strains $\mathrm{M} 07-22$ and $60 \mathrm{P} 3 \mathrm{H} 1$ or to determine the prevalence of $l i c 1$ locus duplication in all strains with licA-licD genes contained purified strain DNA digested with $E c o$ RI and $M f e 1$, respectively. As previously reported by Fox et al [35], strains with duplicate lic1 loci appear on Southern blots as two $M f e 1$ fragments that hybridize

Table 4 Oligonucleotides used in PCR or for DNA sequence analysis

\begin{tabular}{|c|c|c|c|}
\hline Gene & Primer sequences $^{a}$ & Relative position in $\mathrm{Rd}$ & Use \\
\hline \multirow[t]{2}{*}{$\overline{l i c A}$} & $\mathrm{~F}^{\mathrm{b}}$ : GTAGGATTTGTTAAAACTTGCTACAAGCC & 1608693 & probe \\
\hline & R: GGCAATTCCTCTAACAGTTIAAATGCTGCG & 1609579 & \\
\hline \multirow[t]{4}{*}{ licA } & 5'F1: GAATAAATTCATAAGAYTCAGAGCCTTAC & 1608523 & lic1 locus \\
\hline & 5'F2: CAGCTAACCGAGCTTGGGTGAGAAAGTGG & 1608476 & and \\
\hline & mid R: GGCGAAACTCATCGAATACGC & 1609107 & 5'-CAAT- \\
\hline & 3'R: GCCCAAAATACAGCGGACAG & 1609626 & $3^{\prime}$ \\
\hline \multirow[t]{2}{*}{ licB } & F: ATGCGTGGCTATCTCTTTGGCATAC & 1609583 & probe \\
\hline & R: TCATTITTGTTCCCCTITGTAATAAAGTG & 1610461 & \\
\hline \multirow[t]{4}{*}{$l_{i} C B$} & 5'F: GTTATTTGATATAGCGACGATCATTGAGG & 1609316 & lict locus \\
\hline & mid F: CGGATTCGCCTTGGCTATTATTTCTTCTTCG & 1609957 & \\
\hline & mid R: GAGGATATCACTATTTCAGATGACCACCC & 1610091 & \\
\hline & 3'R: GTGTAAATACCCTGTAACAATGACAATATTATCG & 1610628 & \\
\hline \multirow[t]{2}{*}{ licC } & F: ATGAATGCAATCATITIAGCAGCAGG & 1610458 & probe \\
\hline & R: ATGTGGTGATAGTCATCAAGGTTATCC & 1611125 & \\
\hline licC & mid F: CGTATTGATATTGGTTCACTGAATCAACCC & 1610884 & lict locus \\
\hline \multirow[t]{2}{*}{$l i c D$} & F: ATGAAAAAATTGACTCTCAGAG & 1611159 & probe \\
\hline & R: TTACAAAATATACGCTTCTTGAATATG & 1611956 & \\
\hline \multirow[t]{2}{*}{ licD } & 5'F: AATTGGGATACCATTCCGATGG & 1611016 & lict locus \\
\hline & 3'R: AAGGGGCGCAAGAGCAGTTAG & 1612129 & and licD alleles \\
\hline
\end{tabular}

${ }^{\text {a }}$ All oligonucleotides based on DNA sequences from $\mathrm{H}$. influenzae strain Rd or from $\mathrm{H}$. haemolyticus lic1 sequence in this paper to make dot-blot hybridization probes or sequence the lic1 locus, the licD gene alleles, or the licA gene tetranucleotide repeats

${ }^{\mathrm{b}}$ Forward primer begin downstream of licA gene tetranucleotide repeats 
with either $l i c A$ or $l i c D$ gene probes. In our study, we used a $l i c D$ gene probe consisting of combined PCR products representing all three $l i c D$ alleles $\left(l i c D_{I}\right.$ from NT H. influenzae strain 86-028NP and $l i c D_{I I I}$ and $l i c D_{I V}$ from $H$. haemolyticus strains M07-22 and 60P3H1, respectively). All gene probes were labeled, hybridized, and detected as described for dot-blot hybridization, above.

\section{SDS-PAGE and immunoassays}

Whole-cell lysates for SDS-PAGE and Western blotting were obtained by harvesting bacteria in PBS to an O.D. of 1.0, and diluting 4 fold in tricine sample buffer. In the proteinase $\mathrm{K}$ experiments, $10 \mu \mathrm{l}$ of the suspension was incubated with $.5 \mathrm{mg} / \mathrm{ml}$ of proteinase $\mathrm{K}$ at $55{ }^{\circ} \mathrm{C}$ for 2 hours. Untreated or treated bacterial suspension and equal volumes of sample buffer were then heated at $100{ }^{\circ} \mathrm{C}$ for $10 \mathrm{~min}$. and $3 \mu \mathrm{l}$ of preparation were loaded and run on Novex 16\% tricine SDS-PAGE gels and XCell Surelock ${ }^{\mathrm{mw}}$ Mini-Cell apparatus (Invitrogen, Carlsbad, CA) according to the manufacturer's recommendations. Western transfer was performed on a Mini transblot apparatus from Bio-Rad on nitrocellulose membrane (NCM) from Millipore (Bedford, MA). Colony blots were prepared by suspending one colony from the strain of interest in $1 \mathrm{ml}$ of PBS, and plating $100 \mu \mathrm{l}$ of $10^{-6}$ and $10^{-8}$ dilutions on Levinthal agar. Following overnight growth, the colonies were blotted onto NCM discs (Millipore), and the blots were immediately washed in PBS and immunoassayed.

Western and colony-blot immunoassays were performed by first blocking membranes in PBS containing $2 \%$ non-fat dry milk [blotto [56]] for one hour. The blots were then placed in TEPC-15 mAb (Sigma) diluted 1:5000 in blotto for one hour, washed three times with PBS and incubated for one hour in PBS containing 1:5000 goat, anti-mouse IgA antibody conjugated to alkaline phosphatase (Sigma). Following three washes with PBS, a colorimetric reaction was obtained with nitroblue tetrazolium chloride (NBT)/5-bromo-4-chloro3'-indolyphosphate p-toluidine salt (BCIP) substrate (Pierce, Rockford, IL).

\section{Statistical analyses}

All prevalence data were entered in Excel software (Microsoft) in binary form for the presence (which was given a value of 1 ) or absence (which was given a value of 0 ) of any given ChoP-associated genotype. The prevalence ratios of genotypes between NT $H$. influenzae and $H$. haemolyticus were calculated as a ratio of the proportions of genotypes among each species. Chi-square analysis was used to determine the significance of the differences of the genotype associations between species. Statistical analyses were performed with SAS software (version 9.1). Statistical differences in the length of repeat-regions were tested by pair-wise comparisons with the student's $\mathrm{T}$ test.

\section{Acknowledgements}

This work was supported, in part, by Public Health Service grants R03DC006585-01 to KWM and an ARRA 2009 supplement for R01DC0058400751 to JRG and KWM from the National Institute on Deafness and Other Communication Disorders.

\section{Author details}

${ }^{1}$ Department of Pediatrics and Communicable Diseases, University of Michigan, Ann Arbor, Ml 48109, USA. ²Department of Epidemiology, University of Michigan, Ann Arbor, Ml 48109-0244, USA. ${ }^{3}$ Beacon Analytical Systems Inc., Saco, ME 04072, USA.

\section{Authors' contributions}

KWM conceived and directed the study design, performed genetic and immunologic assays, and wrote the manuscript. JX performed genetic assays and did the statistical analyses. CFM and JRG helped in the study design and draft of the manuscript. All authors read and approved the final manuscript.

Received: 17 June 2010 Accepted: 12 November 2010

Published: 12 November 2010

\section{References}

1. Murphy TF, Faden H, Bakaletz LO, Kyd JM, Forsgren A, Campos J, Virji M, Pelton SI: Nontypeable Haemophilus influenzae as a pathogen in children. Pediatr Infect Dis J 2009, 28:43-48.

2. Murphy TF: Respiratory infections caused by non-typeable Haemophilus influenzae. Curr Opin Infect Dis 2003, 16:129-134.

3. Erwin AL, Smith AL: Nontypeable Haemophilus influenzae: understanding virulence and commensal behavior. Trends Microbiol 2007, 15:355-362.

4. Dobrindt U: (Patho-)Genomics of Escherichia coli. Int J Med Microbio/ 2005, 295:357-371.

5. Juliao PC, Marrs CF, Xie J, Gilsdorf JR: Histidine auxotrophy in commensal and disease-causing nontypeable Haemophilus influenzae. J Bacteriol 2007, 189:4994-5001

6. Pettigrew MM, Foxman B, Marrs CF, Gilsdorf JR: Identification of the lipooligosaccharide biosynthesis gene lic2B as a putative virulence factor in strains of nontypeable Haemophilus influenzae that cause otitis media. Infect Immun 2002, 70:3551-3556.

7. Xie J, Juliao PC, Gilsdorf JR, Ghosh D, Patel M, Marrs CF: Identification of new genetic regions more prevalent in nontypeable Haemophilus influenzae otitis media strains than in throat strains. J Clin Microbiol 2006, 44:4316-4325.

8. Kilian M, Mestecky J, Schrohenloher RE: Pathogenic species of the genus Haemophilus and Streptococcus pneumoniae produce immunoglobulin A1 protease. Infect Immun 1979, 26:143-149.

9. Snyder LA, Saunders NJ: The majority of genes in the pathogenic Neisseria species are present in non-pathogenic Neisseria lactamica, including those designated as 'virulence genes'. BMC Genomics 2006, 7:128.

10. McCrea KW, Xie J, LaCross N, Patel M, Mukundan D, Murphy TF, Marrs CF, Gilsdorf JR: Relationships of nontypeable Haemophilus influenzae strains to hemolytic and nonhemolytic Haemophilus haemolyticus strains. J Clin Microbiol 2008, 46:406-416.

11. Takeuchi F, Watanabe S, Baba T, Yuzawa H, Ito T, Morimoto Y, Kuroda M, Cui L, Takahashi M, Ankai A, Baba S, Fukui S, Lee JC, Hiramatsu K: Wholegenome sequencing of Staphylococcus haemolyticus uncovers the extreme plasticity of its genome and the evolution of human-colonizing staphylococcal species. J Bacteriol 2005, 187:7292-7308.

12. Albritton WL: Infections due to Haemophilus species other than $H$. influenzae. Annu Rev Microbiol 1982, 36:199-216.

13. Murphy TF, Brauer AL, Sethi S, Kilian M, Cai X, Lesse AJ: Haemophilus haemolyticus: A human respiratory tract commensal to be distinguished from Haemophilus influenzae. J Infect Dis 2007, 195:81-89. 
14. Kilian M: A taxonomic study of the genus Haemophilus, with the proposal of a new species. J Gen Microbiol 1976, 93:9-62.

15. Olsen I, Dewhirst FE, Paster BJ, Busse H: Family I. Pasteurellaceae Pohl 1981b, $382^{\mathrm{VP}}$ (Effective publication: Pohl 1979, 81). Book Family I. Pasteurellaceae Pohl 1981b, 382VP (Effective publication: Pohl 1979, 81) (Editor ed.^eds.). 2 edition. City: Springer; 2005, 851-856.

16. Takahata S, Ida T, Senju N, Sanbongi Y, Miyata A, Maebashi K, Hoshiko S: Horizontal gene transfer of ftsl, encoding penicillin-binding protein 3 , in Haemophilus influenzae. Antimicrob Agents Chemother 2007, 51:1589-1595.

17. Kuklinska D, Kilian M: Relative proportions of Haemophilus species in the throat of healthy children and adults. Eur J Clin Microbiol 1984, 3:249-252.

18. Kilian M, CR S: Haemophili and related bacteria in the human oral cavity. Arch Oral Biol 1975, 20:791-796.

19. Branson D: Bacteriology and clinical significance of hemolytic Haemophilus in the throat. Appl Microbiol 1968, 16:256-259.

20. Lysenko ES, Gould J, Bals R, Wilson JM, Weiser JN: Bacterial phosphorylcholine decreases susceptibility to the antimicrobial peptide LL-37/hCAP18 expressed in the upper respiratory tract. Infect Immun 2000, 68:1664-1671.

21. Hong W, Mason K, Jurcisek J, Novotny L, Bakaletz LO, Swords WE: Phosphorylcholine decreases early inflammation and promotes the establishment of stable biofilm communities of nontypeable Haemophilus influenzae strain $86-028 \mathrm{NP}$ in a chinchilla model of otitis media. Infect Immun 2007, 75:958-965.

22. Swords WE, Buscher BA, Ver Steeg li K, Preston A, Nichols WA, Weiser JN, Gibson BW, Apicella MA: Non-typeable Haemophilus influenzae adhere to and invade human bronchial epithelial cells via an interaction of lipooligosaccharide with the PAF receptor. Mol Microbiol 2000, 37:13-27.

23. Weiser JN, Pan N, McGowan KL, Musher D, Martin A, Richards J: Phosphorylcholine on the lipopolysaccharide of Haemophilus influenzae contributes to persistence in the respiratory tract and sensitivity to serum killing mediated by C-reactive protein. J Exp Med 1998, 187:631-640

24. Gould JM, Weiser JN: Expression of C-reactive protein in the human respiratory tract. Infect Immun 2001, 69:1747-1754.

25. Claus DR, Osmand AP, Gewurz H: Radioimmunoassay of human C-reactive protein and levels in normal sera. J Lab Clin Med 1976, 87:120-128.

26. Goldenberg HB, McCool TL, Weiser JN: Cross-reactivity of human immunoglobulin G2 recognizing phosphorylcholine and evidence for protection against major bacterial pathogens of the human respiratory tract. J Infect Dis 2004, 190:1254-1263.

27. Moxon R, Bayliss C, Hood D: Bacterial contingency loci: the role of simple sequence DNA repeats in bacterial adaptation. Annu Rev Genet 2006, 40:307-333.

28. Lysenko E, Richards JC, Cox AD, Stewart A, Martin A, Kapoor M, Weiser JN: The position of phosphorylcholine on the lipopolysaccharide of Haemophilus influenzae affects binding and sensitivity to C-reactive protein-mediated killing. Mol Microbiol 2000, 35:234-245.

29. Weiser JN, Love JM, Moxon ER: The molecular mechanism of phase variation of $H$. influenzae lipopolysaccharide. Cell 1989, 59:657-665.

30. Weiser JN, Maskell DJ, Butler PD, Lindberg AA, Moxon ER: Characterization of repetitive sequences controlling phase variation of Haemophilus influenzae lipopolysaccharide. J Bacteriol 1990, 172:3304-3309.

31. De Bolle X, Bayliss CD, Field D, van de Ven T, Saunders NJ, Hood DW, Moxon ER: The length of a tetranucleotide repeat tract in Haemophilus influenzae determines the phase variation rate of a gene with homology to type III DNA methyltransferases. Mol Microbiol 2000, 35:211-222.

32. van Belkum A, Scherer $S$, van Leeuwen W, Willemse D, van Alphen $L$, Verbrugh $\mathrm{H}$ : Variable number of tandem repeats in clinical strains of Haemophilus influenzae. Infect Immun 1997, 65:5017-5027.

33. High NJ, Jennings MP, Moxon ER: Tandem repeats of the tetramer 5'CAAT-3' present in lic $2 A$ are required for phase variation but not lipopolysaccharide biosynthesis in Haemophilus influenzae. Mol Microbiol 1996, 20:165-174.

34. Schweda EK, Richards JC, Hood DW, Moxon ER: Expression and structural diversity of the lipopolysaccharide of Haemophilus influenzae: Implication in virulence. Int J Med Microbiol 2007, 297:297-306.

35. Fox KL, Li J, Schweda EK, Vitiazeva V, Makepeace K, Jennings MP, Moxon ER, Hood DW: Duplicate copies of lic1 direct the addition of multiple phosphocholine residues in the lipopolysaccharide of Haemophilus influenzae. Infect Immun 2008, 76:588-600.
36. Serino L, Virji M: Phosphorylcholine decoration of lipopolysaccharide differentiates commensal Neisseriae from pathogenic strains: identification of licA-type genes in commensal Neisseriae. Mol Microbiol 2000, 35:1550-1559.

37. Leon MA, Young NM: Specificity for phosphorylcholine of six murine myeloma proteins reactive with Pneumococcus $C$ polysaccharide and beta-lipoprotein. Biochemistry 1971, 10:1424-1429.

38. Weiser JN, Shchepetov M, Chong ST: Decoration of lipopolysaccharide with phosphorylcholine: a phase-variable characteristic of Haemophilus influenzae. Infect Immun 1997, 65:943-950.

39. Fleischmann RD, Adams MD, White O, Clayton RA, Kirkness EF, Kerlavage AR, Bult CJ, Tomb JF, Dougherty BA, Merrick JM, McKenney K, Sutton G, FitzHugh W, Fields C, Gocyne JD, Scott J, Shirley R, Liu L, Glodek A, Kelley JM, Weidman JF, Phillips CA, Spriggs T, Hedblom E, Cotton MD, Utterback TR, Hanna MC, Nguyen DT, Saudek DM, Brandon RC, Fine LD, Fritchman JL, Fuhrmann JL, Geoghagen NSM, Gnehm CL, McDonald LA, Small KV, Fraser CM, Smith HO, Venter JC: Whole-genome random sequencing and assembly of Haemophilus influenzae Rd. Science 1995, 269:496-512.

40. Harrison A, Dyer DW, Gillaspy A, Ray WC, Mungur R, Carson MB, Zhong H, Gipson J, Gipson M, Johnson LS, Lewis L, Bakaletz LO, Munson RS Jr: Genomic sequence of an otitis media isolate of nontypeable Haemophilus influenzae: comparative study with $\mathrm{H}$. influenzae serotype d, strain KW20. J Bacteriol 2005, 187:4627-4636.

41. Musser JM, Barenkamp SJ, Granoff DM, Selander RK: Genetic relationships of serologically nontypable and serotype b strains of Haemophilus influenzae. Infect Immun 1986, 52:183-191.

42. Gilsdorf JR, Marrs CF, Foxman B: Haemophilus influenzae: genetic variability and natural selection to identify virulence factors. Infect Immun 2004, 72:2457-2461.

43. Tong HH, Blue LE, James MA, Chen YP, DeMaria TF: Evaluation of phase variation of nontypeable Haemophilus influenzae lipooligosaccharide during nasopharyngeal colonization and development of otitis media in the chinchilla model. Infect Immun 2000, 68:4593-4597.

44. Pang B, Winn D, Johnson R, Hong W, West-Barnette S, Kock N, Swords WE: Lipooligosaccharides containing phosphorylcholine delay pulmonary clearance of nontypeable Haemophilus influenzae. Infect Immun 2008, 76:2037-2043.

45. Pollard A, St Michael F, Connor L, Nichols W, Cox A: Structural characterization of Haemophilus parainfluenzae lipooligosaccharide and elucidation of its role in adherence using an outer core mutant. Can J Microbiol 2008, 54:906-917.

46. Mansson M, Bauer SH, Hood DW, Richards JC, Moxon ER, Schweda EK: A new structural type for Haemophilus influenzae lipopolysaccharide. Structural analysis of the lipopolysaccharide from nontypeable Haemophilus influenzae strain 486. Eur J Biochem 2001, 268:2148-2159.

47. Hogg JS, Hu FZ, Janto B, Boissy R, Hayes J, Keefe R, Post JC, Ehrlich GD: Characterization and modeling of the Haemophilus influenzae core and supragenomes based on the complete genomic sequences of $\mathrm{Rd}$ and 12 clinical nontypeable strains. Genome Biol 2007, 8:R103.

48. Turk DC, May JR: Haemophilus influenzae; its clinical importance. London: English University Press; 1967.

49. Gilsdorf JR, Chang HY, McCrea KW, Bakaletz LO: Comparison of hemagglutinating pili of Haemophilus influenzae type $\mathrm{b}$ with similar structures of nontypeable $\boldsymbol{H}$. influenzae. Infect Immun 1992, 60:374-379, PMC257638.

50. Krasan GP, Cutter D, Block SL, St Geme JW: Adhesin expression in matched nasopharyngeal and middle ear isolates of nontypeable Haemophilus influenzae from children with acute otitis media. Infect Immun 1999, 67:449-454.

51. Sethi S, Evans N, Grant BJ, Murphy TF: New strains of bacteria and exacerbations of chronic obstructive pulmonary disease. $N$ Engl J Med 2002, 347:465-471

52. St Sauver J, Marrs CF, Foxman B, Somsel P, Madera R, Gilsdorf JR: Risk factors for otitis media and carriage of multiple strains of Haemophilus influenzae and Streptococcus pneumoniae. Emerg Infect Dis 2000, 6:622-630

53. Farjo RS, Foxman B, Patel MJ, Zhang L, Pettigrew MM, McCoy SI, Marrs CF, Gilsdorf JR: Diversity and sharing of Haemophilus influenzae strains colonizing healthy children attending day-care centers. Pediatr Infect Dis J 2004, 23:41-46. 
54. Mukundan D, Patel M, Gilsdorf JR, Marrs CF: Pharyngeal colonization characteristics of Haemophilus influenzae and Haemophilus haemolyticus in healthy adult carriers. J Clin Microbiol 2007, 45:3207-3217.

55. Kumar S, Tamura K, Nei M: MEGA3: Integrated software for molecular evolutionary genetics analysis and sequence alignments. Brief Bioinform 2004, 5:150-163.

56. Johnson DA, Gautsch JW, Sportsman JR, Elder J: Improved technique utilizing nonfat dry milk for analysis of proteins and nucleic acids transfer to nitrocellulose. Gene Anal Tech 1984, 1:3-8.

doi:10.1186/1471-2180-10-286

Cite this article as: McCrea et al:: Prevalence of genetic differences in phosphorylcholine expression between nontypeable Haemophilus influenzae and Haemophilus haemolyticus. BMC Microbiology 2010 10:286.

Submit your next manuscript to BioMed Central and take full advantage of:

- Convenient online submission

- Thorough peer review

- No space constraints or color figure charges

- Immediate publication on acceptance

- Inclusion in PubMed, CAS, Scopus and Google Scholar

- Research which is freely available for redistribution

Submit your manuscript at www.biomedcentral.com/submit
Ciomed Central 